\title{
Inclusión financiera y cohesión social en los municipios de México José de Jesús Salazar Cantú*
}

Tecnológico de Monterrey, Campus Monterrey, Departamento de Economía Raymundo Cruz Rodríguez Guajardo

Tecnológico de Monterrey, Campus Monterrey, Departamento de Economía Juvencio Jaramillo Garza

Universidad Autónoma de Nuevo León, Departamento de Administración

(Recibido 13 de Octubre 2017, aceptado 09 de Enero 201\%.)

\begin{abstract}
Resumen
El objetivo del presente estudio es probar el efecto de la inclusión financiera en el nivel de cohesión social en los municipios de México. La metodología contempla la construcción de dos índices de inclusión financiera y la estimación de modelos probit, con información de 2010. Los resultados prueban estadísticamente la relación no lineal, en forma de campana, entre la inclusión financiera y la cohesión social en los municipios mexicanos, igualmente permiten observar la brecha existente entre los niveles de inclusión y aquellos que maximizarían la probabilidad de alcanzar una alta cohesión social municipal. Las principales limitaciones de la investigación fueron la carencia de una teoría consolidada sobre el fenómeno bajo análisis y la falta de información para algunas de las variables consideradas en la estimación. El estudio es el primero que revisa la relación entre la inclusión financiera y la cohesión social a nivel municipal para cualquier país, ambas variables presentes en los planes del actual gobierno mexicano. La inclusión financiera también está significando inclusión social en los municipios mexicanos, consolidar esta estrategia luce promisorio para el pueblo de México.
\end{abstract}

Clasificación JEL: I39, P34, Z13.

Palabras clave: Inclusión financiera, Cohesión social, Desigualdad, Municipios mexicanos.

\section{Financial Inclusion and Social Cohesion in Mexican Municipalities}

\begin{abstract}
The objective of this study is to prove the effect of financial inclusion on the level of social cohesion in Mexican municipalities. The methodology led to the elaboration of two financial inclusion indexes and the estimation of probit models, based on 2010 data. The results statistically prove the non-linear, bell-shaped relation between financial inclusion and social cohesion in Mexican municipalities, and allow to observe the existing gap between inclusion levels and those that would maximize the probability of reaching a high social cohesion at a municipal level. The main limitations of the investigation were the absence of a consolidated theory regarding the analyzed phenomenon, and the lack of information for some of the variables that were considered in the estimation. This study is the first one to elaborate on the relation between financial inclusion and social cohesion at a municipal level for any country, two variables that are present in the Mexican government's current plans. Financial
\end{abstract}

* José de Jesús Salazar Cantú. Ave. Eugenio Garza Sada, 2501 Sur, Col. Tecnológico, Monterrey, Nuevo León, México, C.P. 64849. Tel: 52818358 2000, ext. 4306. Fax 4351. Correo electrónico: jsalazar@itesm.mx 
inclusion also means social inclusion for the municipalities, so the consolidation of this strategy seems promising for the Mexican people.

JEL Classification: I39, P34, Z13.

Key Words: Financial Inclusion, Social Cohesion, Inequality, Mexican Municipalities.

\section{Introducción}

La estrategia "Europa 2020", puesta en marcha en 2010 por los países miembros de la Unión Europea, se enfoca en construir un sistema económico competitivo e inclusivo, que signifique para sus estados miembros: empleo, productividad y cohesión social (European Commission, 2010). En América Latina, el discurso del desarrollo económico, sigue más enfocado en el crecimiento del ingreso, mientras la fragmentación social no es atendida (Sánchez, 2012). Martínez y Estefanía $(2016,20)$ reiteran la necesidad de "un nuevo contrato social", que dé respuesta a las necesidades del creciente número de personas vulnerables, el cual sitúan entre la clase más pobre y la clase media y que ubican como el más numeroso en la región.

"Existe una preocupación generalizada sobre el debilitamiento de la cohesión social en México relacionado, entre otras cosas, con el proceso de urbanización acelerado que se vive en muchas ciudades del país" (México Evalúa y Fundación Hogares, 2015, 7). En el estudio de la cohesión social, diversos autores han mostrado como mayores niveles de ésta, reducen la mortalidad (Inoue, Yorifuji, Takao, Doi y Kawachi, 2013), aumentan el desarrollo humano (Bilbao-Ubillos, 2013), mejoran los índices de satisfacción residencial (Vargas y Merino, 2012) y potencian el crecimiento económico a través del fortalecimiento de la calidad institucional (Easterly, Ritzen y Woolcock, 2006 y CEPAL, 2007 citando a Ocampo, 2004) y de la confianza entre las partes (Granovetter, 2005).

Para Luckmann (1996), el hombre socializado es la condición fundamental para la existencia de la sociedad y, a su vez, la sociedad permite al homo sapiens ser homo sapiente, llevándole a ordenar sus intereses en términos de los intereses de los demás. A este respecto, Berger (1999) advierte como el pluralismo descompone la unidad de los sistemas de valores y presiona hacia un cambio social, orientado a mantener la cohesión social a través de nuevos medios e instituciones. En su postura crítica de la modernidad, Lipovetsky $(2016,300)$ señala: "Vivimos en democracias vaciadas de toda iireligión civilį, de toda fe en grandes proyectos colectivos: la civilización de lo ligero ha vaciado de su sustancia los deberes cívicos y la idea de la obligación ante objetivos sociales superiores".

En el ámbito de la teoría económica, desde mediados del siglo pasado, Gurley y Shaw (1955) ampliaron la explicación del modelo keynesiano de crecimiento económico e incluyeron a la profundidad financiera como causa y a la vez efecto del desarrollo económico. Más recientemente, Bencivenga y Smith (1998) precisaron las circunstancias bajo las cuales se genera el círculo virtuoso establecido por Gurley y Shaw, Makesh y Kuzhuvelil (2014) ubican a la inclusión financiera como determinante de la inclusión social y de un desarrollo más incluyente y Goetzman (2016) muestra cómo, históricamente, las innovaciones financieras han sido determinantes en el desarrollo de la civilización.

Si bien la socialización del individuo puede darse a través de diversos causes, el presente estudio analiza y estima la influencia que la inclusión financiera ejerce 
sobre el nivel de cohesión social entre los municipios de México. Este último concepto, que recientemente fue integrado como indicador de vulnerabilidad social, en el artículo 36 de la Ley General de Desarrollo Social (LGDS) en México.

De acuerdo a cifras del Consejo Nacional de Evaluación de la Política de Desarrollo Social (CONEVAL) (2015), 55.3 millones de habitantes, es decir el 46.2 por ciento de la población en 2014, vivía en algún nivel de pobreza y sólo un 20.5 por ciento de los residentes mexicanos no se encontraban en pobreza o vulnerabilidad social. En el renglón de cohesión social, el CONEVAL sitúa al 51.5 por ciento de los municipios mexicanos en un nivel bajo, en estos municipios, en el año 2010, habitaba el 17.8 por ciento de la población total nacional.

El diagnóstico del cual parte el Plan Nacional de Desarrollo 2013-2018 (PND), describe a un pueblo mexicano cuya principal preocupación es la inseguridad, también reporta que entre 2007 y 2012 el número de homicidios y extorsiones se duplicó, el de secuestros se triplicó y la tasa de victimización alcanzó a casi un cuarto de la población. En respuesta a esta delicada situación, el PND contempla 5 ejes de política pública, dentro del primer eje: "Lograr un México en Paz", encarga la creación del Programa Nacional para la Prevención Social de la Violencia y la Delincuencia (PRONAPRED), a la Comisión intersecretarial para la prevención social de la violencia y la delincuencia (2013), el primero de los dos objetivos generales de este programa, establece el construir comunidades más seguras, cohesionadas e inclusivas (p. 21).

$\mathrm{Si}$ bien la cohesión social es un objetivo prioritario a nivel federal, la inclusión financiera se vislumbra también en la estrategia nacional, como un camino de acceso a dicha cohesión, así, en materia de inclusión social y financiera, el PND, diseñado por el gobierno del presidente Enrique Peña Nieto (2013), dentro del eje: México incluyente, establece en su objetivo 2.2: "Transitar a una sociedad equitativa e incluyente" (p. 116); por su parte, en el eje: "México próspero", el objetivo 4.2 dice: Democratizar el acceso al financiamiento de proyectos con potencial de crecimiento (p. 132).

Las condiciones del desarrollo financiero en México a nivel regional, pueden ser vistas a través de los saldos de la captación estatal en moneda nacional y extranjera realizados por la banca comercial. Información para el año de 2010, obtenida en el Sistema Estatal y Municipal de Bases de Datos (SIMBAD) del INEGI y cálculos propios, permiten observar que el 69.6 por ciento de la captación a nivel nacional se realizó en el Distrito Federal, seguido con un 13.1 por ciento en Nuevo León y 2.9 por ciento en Jalisco. 27 de las 32 entidades federativas mexicanas, recibieron individualmente menos del 1 por ciento del total de captación nacional. Esta realidad habla de una alta concentración de la actividad financiera formal en pocas zonas del país y es sugerente del reto que aún representa la inclusión financiera en México, donde grandes grupos sociales tradicionalmente han sido excluidos de las fuentes de financiamiento para su actividad productiva y social.

Los esfuerzos por aumentar la inclusión financiera en México aún tienen un camino por recorrer, así lo manifiestan Peña, Hoyo y Tuesta (2014), quienes basados en los datos recabados en la Encuesta Nacional de Inclusión Financiera 
2012 del Instituto Nacional de Estadística y Geografía (INEGI), estudian las determinantes de la inclusión en México, en sus conclusiones afirman:

Aunque el $73 \%$ de los municipios en México (con una cobertura de 97\% de la población adulta) tiene al menos un punto de acceso al sistema financiero, esta cobertura es inferior a otros países de referencia en Latinoamérica como Brasil y Chile, e incluso Perú. La oferta alcanzada gracias a cambios regulatorios y a la política pública de inclusión financiera, sólo ha permeado al $38 \%$ de los adultos entre 18 y 70 años quienes tienen algún producto de ahorro o de crédito en instituciones financieras formales. (Peña, Hoyo y Tuesta, 2014: 21)

Entre 2012 y 2015, el porcentaje de adultos que afirmaron contar con al menos un producto de ahorro pasó de 36 a 76.5 por ciento. Por su parte, en el renglón de uso del crédito, el movimiento en dicho lapso fue de 28 a 54.4 por ciento (SHCP, CNBV, INEGI, 2012, e INEGI, CNBV, 2015). En los términos del presente estudio, estos cambios lucen promisorios en materia no sólo de inclusión financiera, sino también social.

La exclusión significa importantes riesgos para el funcionamiento armónico de cualquier sociedad. Fuentes (2008), señala: "La exclusión social implica rechazo, violencia y negación de derechos a veces de la propia existencia de personas, familias o comunidades enteras" (Fuentes, 2008: 187). El mismo autor indica que la actual dinámica del capital global, concentrado en pocas manos, significa la marginación de muchos individuos que no cuentan con las capacidades para participar en los mercados globales y locales, para los cuales se cancela la interacción con el resto de la sociedad, un rasgo de nuestro tiempo al cual considera: "un racismo económico inédito en la historia moderna." (Fuentes, 2008: 192).

A través del uso de métodos estadísticos, la presente investigación prueba la relación no lineal esperada entre el nivel de inclusión financiera existente en las entidades municipales y su efecto en el nivel de cohesión social en los mismos. Esta comprobación es la primera en su tipo realizada para México y resulta de particular importancia, ante el renovado interés por la construcción del tejido social, como medio para acceder a una vida mejor, tanto en lo individual, como en lo colectivo. La investigación aporta elementos de juicio sobre los posibles efectos derivados de las recientes acciones de política pública, incluidas desde el Plan Nacional de Desarrollo (PND) 2007-2012 y continuadas en el PND 20132018, encaminadas a incrementar el acceso y el uso, al y del sistema financiero formal por parte de los residentes mexicanos de todos los estratos sociales, tendencia reciente que no es privativa de México, sino que se extiende también a otros países de ingresos medios y bajos, donde los escenarios socioeconómicos son similares al mexicano.

La presente investigación contribuye al estudio de los efectos del desarrollo financiero sobre la estructura social de dos formas, la primera en términos de realizar una nueva propuesta teórica, donde la cohesión social es influida de manera no lineal por la inclusión financiera; y la segunda al proveer evidencia estadística de esta teoría, para el caso de los municipios de México.

El resto del documento contempla cuatro apartados. A continuación, se plantea el marco teórico y la revisión de literatura referente al fenómeno de estudio. Luego se presenta el modelo propuesto para la estimación econométrica, su estructura basada en la discusión teórico-empírica y su 
especificación, dadas las actuales mediciones de las variables que intervienen en el fenómeno. Finalmente, se interpretan los resultados de las estimaciones y se concluye.

\section{Marco teórico y revisión de la literatura.}

El presente apartado contiene los conceptos básicos de inclusión financiera y cohesión social, aborda los planteamientos teóricos sobre la relación existente entre estas dos variables y finalmente, da cuenta del incipiente estado que guarda la literatura que ha reportado pruebas empíricas en el estudio de la cohesión social.

\subsection{Definiciones}

Si bien se reconoce que no existe una definición generalmente aceptada del concepto de inclusión financiera, en México, el Consejo Nacional de Inclusión Financiera (CONAIF, 2015) la define de la siguiente forma: "El acceso y uso de servicios financieros formales bajo una regulación apropiada que garantice esquemas de protección al consumidor y promueva la educación financiera para mejorar las capacidades financieras de todos los segmentos de la población" (CONAIF, 2015: 1). Dados los fines del presente estudio y su enfoque en el caso mexicano, esta definición es la que se usa para fines de medición y la estimación estadística.

En cuanto al fenómeno de la cohesión social, Chan, To y Chan (2006) advierten que no existe una teoría de base sobre el mismo, ni una definición consensada del propio concepto, su propuesta definicional es la siguiente:

Es un estado de cosas en relación tanto a las interacciones verticales como horizontales entre miembros de la sociedad, que se caracteriza por un conjunto de actitudes y normas que incluyen la confianza, el sentido de pertenencia y la voluntad de participar y ayudar, así como sus manifestaciones conductuales (Chan et al., 2006: 290)

En teoría social es común referir el sentido de cohesión social como la capacidad de la comunidad para reproducirse a sí misma en el largo plazo, siendo Durkheim (1993) y Puntman (2000) dos de sus principales referentes.

Cohesión social y capital social son conceptos que parecen traslaparse y llegan a ser empleados como sinónimos, la Comisión Económica para América Latina y el Caribe, CEPAL los distingue de la siguiente forma:

Una primera noción cercana a la de cohesión es la de capital social, entendido como patrimonio simbólico de la sociedad en términos de la capacidad de manejo de normas, redes y lazos sociales de confianza, que permiten reforzar la acción colectiva y sentar bases de reciprocidad en el trato, que se extienden progresivamente al conjunto de la sociedad (CEPAL, 2015: 15). La cohesión social se define como la dialéctica entre mecanismos instituidos de inclusión y exclusión sociales y las respuestas, percepciones y disposiciones de la ciudadanía frente al modo en que ellos operan. (CEPAL, 2015: 16) señala:

Oxoby (2009), al referir capital social, cohesión social e inclusión social,

El capital social es un sacrificio por parte del individuo (tiempo, esfuerzo, consumo) hecho en un esfuerzo por promover la cooperación con otros. La cohesión social es una característica de la sociedad, la cual depende del capital 
social acumulado. Y la inclusión social es un aspecto de cómo uno percibe su acceso a instituciones y recursos en el ámbito de la toma de decisiones. (Oxoby, 2009:5).

La ausencia de una definición consensada de cohesión social planea un reto también a la hora de su medición. En México, el CONEVAL empezó a generar este indicador a raíz del mandato expreso de la Cámara de Diputados (2013), que llevó a la modificación del artículo 36 de la LGDS, el cual contempla la forma de medición de la pobreza, señalando la inclusión de un indicador de cohesión social. En la sección de metodología se expondrán los elementos de medición del CONEVAL.

Si bien el concepto de cohesión social tiene sus raíces en la sociología y la psicología social, atendiendo a la disponibilidad de información a nivel municipal en México, el presente estudio sigue la óptica del Consejo Nacional de Evaluación de la Política de Desarrollo Social (CONEVAL), cuyo enfoque correspondería más al de la sociología neo-positivista, y a la idea de Almeida y Sánchez (2014), ya que visualiza la dimensión geográfica y de diferencia de ingresos, como partes preponderantes o al menos representativas, del nivel de cohesión social en el nivel municipal.

A continuación, se abordan las ideas que enmarcan la relación teórica esperada entre inclusión financiera y cohesión social.

\subsection{Teoría}

Ante la ausencia de una teoría de base que refiera la relación esperada entre la inclusión financiera y la cohesión social, en este apartado se revisan algunas de las teorías más cercanas a la explicación del fenómeno.

En la teoría económica, Gurley y Shaw (1955) muestran que el desarrollo económico y el financiero se determinan mutuamente. Por su parte, Bencivenga y Smith (1998) describen ciertas condiciones, bajo las cuales el sistema financiero puede o no llevar a un desarrollo económico estable. En este mismo sentido, Goetzman $(2016,14)$ señala: "Las relaciones financieras se han convertido en medios importantes, a través de los cuales las economías se unen en una compleja civilización global".

Desde el modelo de Bencivenga y Smith (1998), el cual observa dos posibles explicaciones al bajo efecto del desarrollo financiero en el desarrollo económico de un país, podría decirse, que, en el caso mexicano, los altos costos de intermediación financiera han hecho prohibitivo el uso del sistema financiero a amplios grupos de la sociedad mexicana. La reducción paulatina de estos costos, ya sea por los cambios en la regulación, o por el mayor acceso a tecnologías de información y comunicación más ágiles y cada día más disponibles, así como la creciente competencia en diversas áreas de la intermediación financiera, entre otras causas, significa un paso hacia una mayor y mejor distribuida profundidad del sistema financiero, aumentando la probabilidad de generar el círculo virtuoso previsto en el modelo de Gurley y Shaw. Tan importante es este proceso, que su ausencia por décadas o incluso siglos en el caso mexicano, es expuesta por Acemoglu y Robinson (2013, 48-52) como una de las principales razones de la amplia diferencia en el progreso económico experimentado por los Estados Unidos, versus el de México durante los siglos XIX y XX, en los cuales EE.UU. logró tanto un importante crecimiento en su 
ingreso como reducción en el nivel de desigualdad, mientras que en México el crecimiento fue más lento y la desigualdad prácticamente se quedó igual.

Como muestran formalmente Bencivenga y Smith (1998), en la medida que aparece y crece la intermediación financiera, en equilibrio general se esperaría una producción más intensiva en el uso del capital, lo que suele hacerla más eficiente y productiva. Por su parte, como lo demuestran Greenwood y Jovanovic (1990) el mismo incremento en la intermediación también llevará, en sus etapas más avanzadas, a la reducción en la desigualdad de ingresos y entonces a una mayor cohesión social.

Desde la sociología y considerando la condición geográfica de asentamientos humanos, Timasheff (2006), observa el principio teórico del menor esfuerzo de Zipf (1949), quien desde el neo-positivismo explica la distribución poblacional en diversas comunidades. De acuerdo a este principio, cuando las materias primas para el trabajo son pocas, las familias decidirán vivir en pequeñas comunidades aisladas y cercanas a dichas materias primas, de otra forma, tenderán a concentrarse cerca de los lugares de trabajo. En esta óptica, la existencia de polos poblacionales aislados, podría llevar a una menor cohesión. En México, el proceso de urbanización ha llevado a que, el 80 por ciento de la población resida en las ciudades (Instituto Mexicano para la Competitividad, IMCO, 2014).

El estudio de la cohesión social, puede también entenderse desde la sociología funcionalista, para la cual, las partes del sistema social desempeñan funciones esenciales para la subsistencia del todo, con lo que son interdependientes. En la medida que estas partes están integradas, el sistema prevalece, y entra en declive cuando éstas se desvinculan, pero el nivel de unión entre unas y otras puede depender también de la cohesión misma experimentada hacia dentro de cada elemento del todo, con lo que el fenómeno no necesariamente es lineal, así lo advierten las ideas de Granovetter (1973).

Los efectos propiciados por los cambios en el grupo social, ya sea éste la familia, la comunidad o la sociedad, implican también cambios en la manera de pensar y actuar de las personas que conforman el grupo (Luckmann, 1996). Garrido y Álvaro (2007) refieren cómo, desde fines del siglo XIX, se empieza a dar el estudio del comportamiento de las masas en escritos de Gustave Le Bon, para el cual, entidades psicológicas supraindividuales surgen de la vinculación-desvinculación entre los individuos, afectando incluso su identidad y pertenencia a un grupo. Para Fromm (2010), la reacción psicológica del grupo social, ante los eventos por los que transita, dependerá del carácter social, común denominador de la estructura del carácter de la mayoría de miembros de dicho grupo, que, a su vez, determina la capacidad de adaptación de éste. El mismo autor, ejemplifica como los principios de esfuerzo incesante y religión del éxito, dirigidos al campesino e indígena mexicano, si bien entendidos, no hallaron respuesta favorable y siguen significando abismos entre los mismos y sus connacionales.

En el estudio del fenómeno de comunidad, Almeida y Sánchez (2014), refieren que los tiempos actuales no son propicios para reforzar los lazos comunitarios, señalan: En el mundo globalizado, el rasgo más distintivo que distingue al sistema económico-político y sociocultural en el que estamos inmersos es la desigualdad (Almeida et al., 2014: 168). La cohesión se ve 
debilitada, ante las diferentes expresiones de desigualdad imperantes entre los subgrupos de la sociedad.

La demanda cada vez mayor por pluralidad como objetivo social, ha venido también a descomponer los sistemas de valores, ante ello, las sociedades van reaccionando para restablecer la cohesión social, así lo considera Berger (1999). El fenómeno ha coincidido con el aumento de los desarrollos tecnológicos enfocados en la información y comunicación, a través de los cuales están cambiando las formas de interacción social. En el marco del presente estudio, se considera que estos cambios tecnológicos recientes, por un lado han dado la pauta para una mayor inclusión y cohesión social, ya que aumentan la disponibilidad del recurso financiero para poner en marcha ideas productivas a cada vez más agentes económicos, muchos de ellos tradicionalmente excluidos de esta posibilidad, pero por otro, también plantean la posibilidad de una mayor autonomía individual, la cual se generaría en una segunda etapa, luego de haber logrado la inclusión, lo cual podría operar en contra de la propia cohesión social inicialmente dada, de ahí la expectativa de una relación en forma de U invertida.

Dado este marco teórico, se llega a la tesis de que, dado lo demás constante, la inclusión financiera aumenta la cohesión social. Por su parte, también se postula que la influencia de la inclusión financiera sobre la cohesión social sigue la forma de una U invertida, la cual es representada en la gráfica 1.

Gráfica 1. Inclusión financiera y cohesión social.

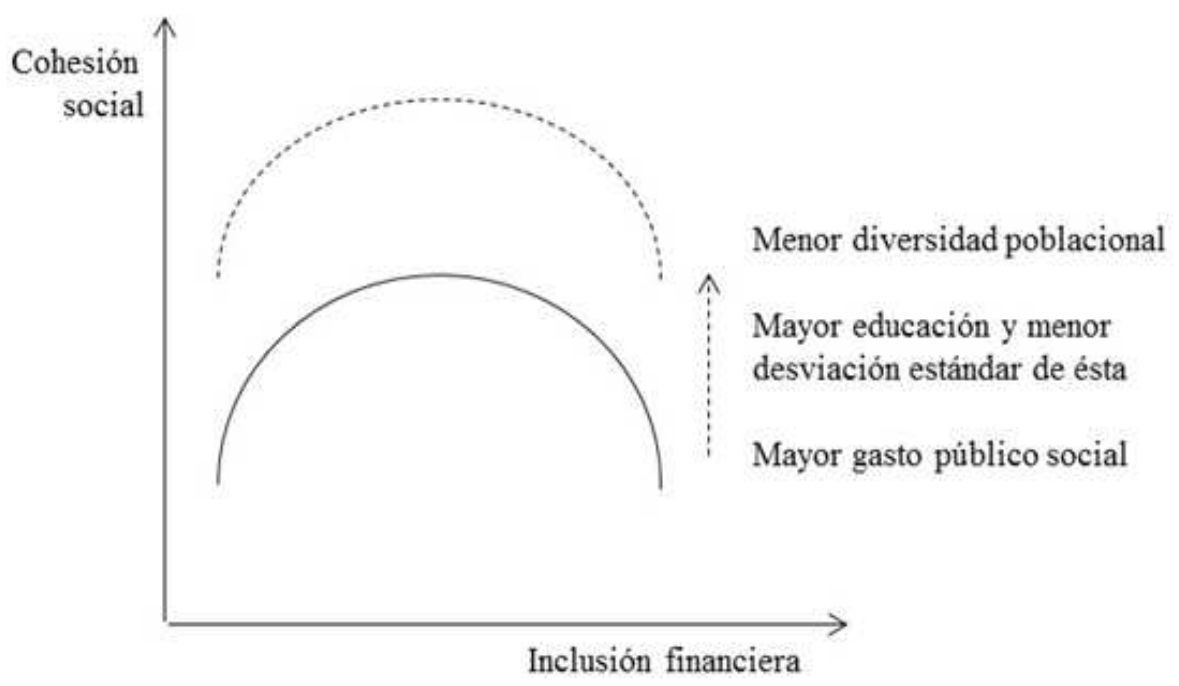

Como puede verse en la gráfica 1, la influencia de la inclusión financiera en la cohesión social se espera sea en forma de U invertida, la cual en una primera etapa sigue el patrón esperado de acuerdo a las ideas de Gurley y Shaw (1955), Bencivenga y Smith (1998) y Makesh y Kuzhuvelil (2014), donde, dado lo demás constante, una mayor inclusión financiera aumenta el grado de cohesión social. Por su parte, el presente estudio propone que en la medida que la cohesión aumenta, nuevos incrementos de la inclusión financiera cada vez impactarían 
en menor medida a la cohesión social, llegando incluso a reducirla, cuando una mayor inclusión financiera convierte a los agentes micro en unidades cada vez más autónomas. La relación esperada entre ambas variables también se ve influenciada por otros determinantes, como son: el nivel de ingresos, la existencia de polos poblacionales, la participación en redes sociales, la educación, la diversidad étnica y la propia intervención del gobierno en el sistema social, estas variables, también presentes en la literatura empírica, implican desplazamientos de la curva hacia lugares distintos en el semiplano. Por ejemplo, la inexistencia de polos o un mayor nivel educativo, llevaría a un desplazamiento de la función hacia arriba, mientras una menor participación en redes sociales o la mayor diversidad etnolingüística la desplazaría hacia abajo. El efecto del ingreso puede ser también no lineal, un aumento del ingreso podría significar inicialmente una mayor posibilidad de integración social, pero luego de cierto umbral, podría significar el aislamiento y autonomía, por su parte, el efecto del ingreso sobre la cohesión social podría darse de manera indirecta a través de su impacto en su distribución, fenómeno estudiado por Kuznets (1955) quien postuló una función en forma de U invertida entre el ingreso y su distribución. En este caso, el efecto sería entonces de U, entre la inclusión financiera y la cohesión social, esta última que contempla en su medición, de acuerdo a CONEVAL (2015), la desigualdad de ingresos.

De acuerdo a Kempson, Whyley, Caskey y Collard (2000) se esperaría que un mayor índice de diversidad poblacional etnolingüística llevara también a mayor desigualdad de ingresos y entonces una menor cohesión social, por su parte, de acuerdo a Clarke, Xu y Zou (2006), los mayores gastos públicos per cápita harían esperar una menor desigualdad de ingresos y con ello una mayor cohesión social, estos autores señalan el objetivo general de los gobiernos en lograr mayor igualdad. En lo tocante a la educación, Gradstein y Justman (2002), muestran como ésta es una fuerza que lleva a la cohesión social.

Si bien el CONEVAL ha medido el grado de cohesión social a nivel municipal en México, para este mismo país también hay una propuesta de medición de Boltvinik (2010) basada en cifras relativas al crimen, donde mayor crimen es asociado con menor cohesión social. Esta forma de medición sigue la línea de Granovetter (2005), según la cual, la variable de crimen estaría relacionada con los tres canales de influencia entre la estructura social y la actividad económica. En la revisión de literatura, también se encontró la reciente propuesta de un índice de cohesión social a nivel vecinal, el cual ha sido desarrollado por las organizaciones México Evalúa y Fundación Hogares (2015), la visión de este último es más cercana a la concepción de cohesión social de Chan et al. (2006) y al concepto de comunidad de Almeida y Sánchez (2014) y no tanto con la sociedad, refiere más al barrio y no al municipio, al nivel micro, no al macro.

Sobre el índice propuesto por Boltvinik (2010) para medir la cohesión social a nivel municipal, Foster (2010), indica: "No hay razones para suponer que la actividad criminal reportada reflejará la actividad criminal real, ni para pensar que ésta es un indicador particularmente adecuado de la cohesión social, aun si tal actividad es medida en forma correcta" (Foster, 2010: 379).

Al realizar la aplicación de su índice vecinal, México Evalúa y Fundación Hogares (2015) tomaron como casos de estudio 4 conjuntos habitacionales de vivienda de interés social, uno al norte, dos del centro y uno del sur de México. 
En una escala estandarizada a valores de 0 a 10 , los cuatro conjuntos alcanzaron un índice de poco más de 5 , donde el subíndice más bajo fue el referente a participación comunitaria. Este estudio es pionero en México e ilustra el interés de la sociedad civil organizada por el fenómeno de la cohesión social. Por su parte, los pocos datos existentes hasta ahora sobre los niveles de cohesión a este nivel micro, no son suficientes aún para probar la forma en que las relaciones entre la cohesión social micro y macro se están dando en México y la influencia de éstas en aspectos económicos, como serían el crecimiento del ingreso y la inclusión financiera, entre otros.

Hasta donde se pudo encontrar en la revisión de literatura del presente estudio, la investigación empírica, relativa al fenómeno de la inclusión financiera y su influencia en la cohesión social es incipiente, ello es un reflejo también de lo reciente del concepto de inclusión financiera, de sus formas de medición y principalmente, de la falta de teorías consolidadas que expliquen sus causas y efectos.

Desde el ámbito de la economía, en la investigación más relacionada con el fenómeno que aborda el presente estudio, Arcand, Berkes y Panizza (2012) y Ratna, Cihák, NDiaye, Barajas, Bi, Ayala, Gao, Kyobe, Nguyen, Saborowski, Svirydzenka y Reza, (2015) analizan la relación no lineal entre profundidad financiera y crecimiento económico. En ambos trabajos se encuentra una relación en forma de campana donde, en niveles relativamente bajos de profundidad financiera, la pendiente es positiva. No obstante, se llega a un máximo después del cual a mayor profundidad financiera hay menor crecimiento económico. Arcand et al. (2012), utilizan información a nivel de países para el período 1960 2010. Por su parte, Ratna et al. (2015) usan una muestra de 128 países para el periodo 19802013. En ambos estudios, se prueban dos razones que explican esta relación inversa entre profundidad financiera y crecimiento económico. La primera es la mayor volatilidad económica y aumento de la probabilidad de crisis e inestabilidad macroeconómica, y la segunda es la asignación ineficiente de los recursos, ya que el capital humano se retira de los sectores productivos para irse al sector financiero.

$\mathrm{Al}$ indagar sobre trabajos previos realizados para México, Venegas, Tinoco y Torres (2008), Rodríguez y López (2009), así como Zavaleta y Urbina (2011), han estudiado el desarrollo financiero en México y su efecto en el crecimiento de este país. Mientras, Venegas et al. (2008), encuentran que el desarrollo financiero no ha influido en el crecimiento del producto interno bruto mexicano, Rodríguez y López (2009) y Zavaleta y Urbina (2011), empleando diferentes formas de medir el desarrollo del sistema financiero mexicano, obtienen un efecto positivo de éste hacia el crecimiento económico del país. Un estudio etnográfico, realizado entre familias de estratos pobres en Bangladesh, India, México y Sudáfrica, Collins, Morduch, Rutherford y Ruthven (2011), los autores encuentran que la disponibilidad de crédito suele ser menor para las personas pobres, por su parte, los esquemas de préstamo en instituciones formales, se adaptan menos a la volatilidad característica de los ingresos de este grupo, observan plazos menos flexibles que los que demandaría su exposición al riesgo y cuando existe, suele exhibir tasas de interés más altas, comparadas con aquellas a las que tiene acceso el público no pobre. Así, concluyen que la inclusión financiera no sólo requiere de la posibilidad de acceso, sino también de nuevos esquemas 
de financiamiento que reconozcan este perfil de agentes. El trabajo de campo de este mismo estudio, deja en claro, la importancia que tienen las relaciones vecinales para lograr el acceso a pequeños préstamos informales, monetarios y/o en especie, ante imprevistos.

Para el caso de los municipios de México, Salazar, Jaramillo y Álvarez (2015), confirman la relación, en forma de U invertida, entre el nivel de inclusión financiera y el coeficiente de distribución del ingreso, planteada por Greenwood y Jovanovic (1990). Entre las líneas de investigación que plantean dichos autores, señalan la necesidad de estudiar la relación entre la inclusión financiera y la cohesión social, este último concepto, que en su estudio se plantea como un objetivo paralelo al de la igualdad de ingresos, pero que también observa la noción de cercanía y convivencia entre los residentes mexicanos. El presente estudio da seguimiento a esta línea de investigación.

A continuación, se presentan la metodología y los datos que serán empleados en la comprobación de la relación existente entre la inclusión financiera y la cohesión social en los municipios de México.

\section{Metodología y datos}

El presente estudio estima la relación entre las variables de inclusión financiera y cohesión social controlando para variables concomitantes.

Partiendo de las propuestas teóricas de Gurley y Shaw (1955), Bencivenga y Smith (1998) y Makesh y Kuzhuvelil (2014), se probará la influencia de la inclusión financiera en la cohesión social, relación expresada en la ecuación 1. Donde la inclusión financiera medida por el lado del acceso al sistema $\left(\right.$ iia $\left._{j}\right)$ o en cuanto al uso del mismo $\left(i i u_{j}\right)$, determina a la cohesión social del $j$-ésimo municipio $\left(c s_{j}\right)$.

$$
c s_{j}=f\left(i i a_{j} \text { о } i i u_{j}, \cdots\right)
$$

Donde:

$c s_{j}$ : Cohesión social;

$i i a_{j}$ : Índice de inclusión financiera según acceso al sistema financiero formal; e

$i i u_{j}$ : Índice de inclusión financiera según el uso del sistema financiero formal.

Tal como establecen Chan, et al. (2006), no existe una teoría consensada de cohesión social, sino que ésta se encuentra en discusión. La revisión de literatura del presente estudio permitió también ver que hay diversos niveles a los cuales se entiende el fenómeno de la cohesión social: Entre los países; hacia dentro de los países; entre las regiones de un mismo país; en el ámbito del barrio; y entre las personas más allegadas. En cada uno de los niveles, la cohesión puede obedecer a diferentes aspectos, por su parte, en todos ellos refiere el sentido de unión que hay entre las partes. En la aproximación empírica del presente estudio, se analiza la cohesión social entre las regiones de México, definidas éstas desde su concepción geopolítica, como los 2,456 municipios que conformaban al país en el año de 2010.

En el análisis se emplean los datos de cohesión social de CONEVAL (2015, b). En el sentido de CONEVAL, cohesión social es una variable binaria que clasifica a cada municipio mexicano como de baja (0) o alta (1) cohesión $\left(c s_{j}\right)$. De acuerdo a CONEVAL (2015), la construcción de este indicador contempla 
cuatro elementos: a) El coeficiente de Gini; b) La razón de ingresos entre el grupo en pobreza extrema y el no pobre ni vulnerable; c) La existencia de polos poblacionales; y d) La percepción de participación en redes sociales.

En cuanto a la inclusión financiera, en el presente estudio se sigue la definición del CONAIF (2015), la cual contempla tanto el lado del acceso al sistema financiero formal, como el uso del mismo. De acuerdo a esta concepción, se construyen dos índices sumativos, como variables que permiten aproximar el nivel de inclusión financiera municipal. Se emplean datos de la Comisión Nacional Bancaria y de Valores (2015) referentes a tres diferentes puntos posibles de acceso: Sucursales bancarias, cajeros automáticos y corresponsales bancarios, por cada 10,000 habitantes, con ellos se construye el índice de acceso (iia). De la misma base, se consideran los datos de 8 tipos distintos de contratos entre unidades del sistema financiero y el público por cada 10,000 habitantes, para construir un índice de uso (iiu). Esta forma de medición de ambos índices es propuesta y empleada en Salazar et al. (2015).

Como fue expuesto en el apartado de marco teórico y de revisión de la literatura, desde la sociología y la psicología social, se relaciona a la cohesión social con diversos aspectos de los grupos poblacionales, entre ellos: la influencia que representa el pertenecer a alguna etnia, el nivel de ingresos y de educación de los elementos del grupo, así como el nivel de intervención del gobierno en la sociedad, expresado a través de la variable de gasto público municipal, aspectos que influyen en el carácter social del grupo. En el presente estudio, dichos elementos serán considerados variables de control, que al ser contempladas dentro del modelo general, expresado en (1), llevarían a la siguiente relación funcional (2):

$$
c s_{j}=f\left(i i a_{j} \mathrm{oiiu}_{j}, i n g_{j}, e d u_{j}, i d e l_{j}, \text { gpubp }_{j}, \cdots\right)
$$

Al pasar la ecuación (2) a su forma explícita, se construye la ecuación (3), misma que será utilizada en la estimación para comprobar la posible influencia de la variable de inclusión financiera sobre la cohesión social. Dada la relación no lineal esperada entre la inclusión financiera y la cohesión social, en la ecuación (3) se incluye la variable explicativa de inclusión financiera tanto en niveles, como en su término cuadrático, lo mismo se hace con la de ingreso, según la expectativa teórica basada en Kuznets (1955), de una relación no lineal entre éste y la distribución del ingreso y entonces con el nivel de cohesión social, según la medición de esta última del CONEVAL.

$$
c s_{j}=a+\beta_{1} i f_{j}+\beta_{2} i f_{j}^{2}+\beta_{3} i n g_{j}+\beta_{4} i n g_{j}{ }^{2}+\beta_{5} e d u_{j}+\beta_{6} i d e l_{j}+\beta_{7} g p u b p_{j}+\varepsilon
$$

Donde: $c s_{j}$ : Cohesión social;

$i f_{j}$ : Nivel de inclusión financiera, por acceso (iia) o por uso (iiu);

$i_{\text {ing }}$ : Ingreso per cápita medio;

$e d u_{j}$ : Nivel medio de educación de los jefes de hogar;

$i d e l_{j}$ : Índice de fraccionalización etnolingüística;

gpubp $_{j}$ : Gasto público per cápita; y $\varepsilon$ : Término de error. 
De acuerdo al marco teórico que plantea una causalidad de la inclusión financiera sobre la cohesión social y la revisión de literatura, se espera que: $\beta_{1}>0, \beta_{2}<0, \beta_{3}<0, \beta_{4}>0, \beta_{5}>0, \beta_{6}<0$ y $\beta_{7}>0$. En el cuadro 1 , se presentan las variables y sus fuentes:

Cuadro 1. Las variables, su abreviación, descripción y fuentes.

\begin{tabular}{|c|c|c|}
\hline Vars. & Descripción & Fuente \\
\hline Csd & $\begin{array}{l}\text { Cohesión social como variable binaria. Se } \\
\text { considera de alta cohesión social (1) a los } \\
\text { municipios con polo derecho (de baja } \\
\text { marginación) o sin polo y de baja cohesión } \\
\text { social }(0) \text { a los municipios polarizados o } \\
\text { con polo izquierdo (de alta marginación). }\end{array}$ & $\begin{array}{l}\text { Consejo Nacional de Evahuación de la } \\
\text { Politica de Desarrollo Social } \\
\text { (CONEVAL) } \\
\text { http://www.coneval.gob.mx/Medicion/ } \\
\text { Paginas/Cohesion_Social.aspx }\end{array}$ \\
\hline $\begin{array}{l}\text { Csb } \\
\text { Iia }\end{array}$ & $\begin{array}{l}\text { Cohesión social, Boltvinik (2010). } \\
\text { Indice de inclusión financiera según acceso. } \\
\text { Suma el promedio de: Sucursales bancarias, } \\
\text { corresponsales bancarios y cajeros } \\
\text { automáticos, por cada } 10,000 \text { habitantes. }\end{array}$ & $\begin{array}{l}\text { Construcción con datos de SIMBAD. } \\
\text { Comisión Nacional Bancaria y de } \\
\text { Valores (CNBV) } \\
\text { http://portafoliodeinformacion.cnbv.go } \\
\text { b.mx/estudios/Paginas/default.aspx }\end{array}$ \\
\hline Iiu & $\begin{array}{l}\text { Índice de inclusión financiera según uso. } \\
\text { Suma cuentas de cheques, de ahorro, de } \\
\text { nómina, de depósitos a plazo, número de } \\
\text { tarjetas de crédito y de débito, préstamos } \\
\text { hipotecarios y grupales }\end{array}$ & $\begin{array}{l}\text { Comisión Nacional Bancaria y de } \\
\text { Valores (CNBV) } \\
\text { http//portafoliodeinformacion.cnbv.go } \\
\text { b.mx/estudios/Paginas/default.aspx }\end{array}$ \\
\hline Edu & $\begin{array}{l}\text { Promedio de años de educación del grupo } \\
\text { de jefes de hogar a nivel municipal. }\end{array}$ & $\begin{array}{l}\text { Comisión Nacional Bancaria y de } \\
\text { Valores (CNBV) } \\
\text { http://portafoliodeinformacion.cnbv.go } \\
\text { b.mx/estudios/Paginas/default.aspx }\end{array}$ \\
\hline Ing & $\begin{array}{l}\text { Proxy del ingreso per cápita municipal } \\
2010 \text {. Se emplea una estimación realizada } \\
\text { siguiendo el método de Ibarra y Varella } \\
\text { (2006) el cual parte del pib estatal y es } \\
\text { imputado a través del nivel de empleo. Los } \\
\text { datos se expresan en miles de pesos } \\
\text { constantes de } 2003 \text {. } \\
\text { Índice de fraccionalización etnolingüística, } \\
\text { visto como indice de diversidad } \\
\text { poblacional. }\end{array}$ & $\begin{array}{l}\text { Olvera, Giselle, Collado, Nashtenka, } \\
\text { Martínez, A lejandra y Bueno, Mariela } \\
\text { (2014). Diversidad poblacional y } \\
\text { crecimiento económico en México. } \\
\text { Revista Estudiantil de Economía, } 6(1) \text {, } \\
1-20 \text {. (Base de datos de libre acceso en } \\
\text { https:/drive.google.com, en el archivo: } \\
\text { base de datos sem (1), usuario: } \\
\text { sem.mex.ad13, contraseña: diversidad) }\end{array}$ \\
\hline $\mathrm{Pfc}$ & Fioce & INEGI-SIMBAD \\
\hline Gpubp & $\begin{array}{l}\text { Gasto público per cápita municipal del } \\
2010 \text {, en pesos corrientes. }\end{array}$ & $\begin{array}{l}\text { Instituto Nacional para el Federalismo } \\
\text { y el Desarrollo Municipal (INAFED). } \\
\mathrm{http} / / \text { www.snim.rami.gob.mx/ }\end{array}$ \\
\hline
\end{tabular}

Fuente: Elaboración propia.

Dado que la muestra utilizada en esta investigación está altamente balanceada entre ceros y unos para la variable dependiente $(51.5 \%$ y $48.5 \%$, respectivamente), "se esperan resultados muy similares al utilizar los modelos de probabilidad probit y logit binarios" (Greene, 2012: 734-735). Adicionalmente, conforme a IDRE-UCLA (2015), la selección de uno de estos dos modelos "depende principalmente de las preferencias individuales". Con base en lo anterior, el método de estimación que se emplea en el presente estudio al realizar inferencia estadística sobre el concepto de cohesión social medido en forma binaria, es el de probit. La variable dependiente es la binaria de cohesión social, la cual será explicada por el nivel de inclusión financiera (iia, iiu), dado el grupo 
de variables de control (ing, edu, idelygpubp). A continuación, se presentan los resultados tanto de la parte descriptiva como de inferencia estadística.

\section{Resultados}

El cuadro 2 presenta la estadística descriptiva de las variables que intervienen en la comprobación. En 2010 se contaban 2456 municipios en México, de la información encontrada en las fuentes de libre acceso, se perdieron 16 observaciones, donde los datos que permitieron el cálculo de las variables de inclusión financiera rebasaban los límites de medición esperados, sin haber encontrado alguna explicación precisa de este hecho. Por su parte, tanto en la variable de diversidad poblacional (idel), como en la de gasto público per cápita (gpubp), el número de observaciones difiere del total de municipios debido a que no existen datos para algunos de ellos.

Cuadro 2. Estadística descriptiva.

\begin{tabular}{|c|r|rr|r|r|r|}
\hline & No. obs. & Media & Mediana & Desv. est. & \multicolumn{1}{l|}{ Mín. } & \multicolumn{1}{c|}{ Máx. } \\
Csd & 2440 & 0.48 & 0 & 0.50 & 0 & 1 \\
\hline Csb & 2118 & -0.29 & 0.0134 & 1.16 & -8.83 & 1 \\
\hline Iia & 2440 & 3.07 & 1.49 & 4.62 & 0 & 42.76 \\
\hline Iilu & 2440 & 6135.52 & 574 & 10407.10 & 0 & 77175.3 \\
Ing & 2440 & 79.08 & 71.90 & 49.30 & 9.68 & 1346.2 \\
\hline Edu & 2440 & 5.55 & 5.29 & 1.75 & 1.33 & 13.13 \\
\hline Idel & 2417 & 0.12 & 0.03 & 0.16 & 0 & 0.50 \\
\hline gpubp & 2102 & 3959.62 & 3277.91 & 2441.97 & 744.31 & 33562.7 \\
Pfc & 2118 & 0.96 & 0.73 & 0.86 & 0 & 7.26 \\
\hline
\end{tabular}

Fuente: Elaboración propia.

El grupo de municipios con baja cohesión, según la medida de CONEVAL, es ligeramente mayor al de alta cohesión, por ello la media de csd se acerca a un medio. Por su parte, al considerar esta variable calculada según la propuesta de Boltvinik (2010), csb, la cual considera la proporción que en la mediana nacional del número de procesados del fuero común por cada mil habitantes, representa la diferencia entre dicha mediana y el número de procesados en cada municipio, la mediana de este indicador tiene un número positivo, cercano a cero, el cual indicaría que más de la mitad de los municipios (1073 de 2118, 50.7 por ciento) cuentan con cohesión media, alta o muy alta, dicho resultado es similar al que arroja la variable binaria de CONEVAL, ello aumentó la confianza en el uso del índice construido y publicado por CONEVAL.

La inclusión financiera muestra una mediana nacional de 1.5 puntos de acceso y 574 cuentas, por cada 10,000 habitantes en 2010. También se encontraron 231 municipios donde ambos índices tomaron el valor de cero, la mayoría de ellos situados en el estado de Oaxaca.

El promedio municipal del PIB per cápita de 2010, a precios de 2003, fue de casi 80 mil pesos. Por su parte, la diferencia de este indicador entre los municipios de menor y mayor monto fue de casi 141 veces. La diferencia entre el nivel educativo medio de los jefes de hogar en el municipio con menor número de años cursados y el de mayor número, fue de 10 veces, la media nacional de esta variable fue de 5.6 años, lo que indica un nivel promedio menor al de la educación primaria completa. 
En los municipios con mayor diversidad etnolingüística, la mitad de la población habla al menos dos lenguas. Por su parte, en la mitad de los municipios mexicanos, la probabilidad de encontrar a alguien que hable una lengua indígena es menor al 3 por ciento.

El gasto público medio por persona en 2010, fue de 3959.6 pesos, es decir, el equivalente a 2.4 veces el salario mínimo general mensual, o lo que es lo mismo, un promedio de 0.85 dólares diarios por persona, considerando el tipo de cambio de junio de ese año. Finalmente, la media y mediana de procesados del fuero común por cada 1000 habitantes son cercanas a 1 y en 69 municipios el valor fue de 0 . A continuación, en el cuadro 3 se presentan los resultados de la estimación de (3).

Cuadro 3. Estimación del modelo probit. Variable dependiente: csd.

\begin{tabular}{|c|c|c|c|c|}
\hline Ecuación & 1 & & 2 & \\
\hline Método & Probit & & Probit & \\
\hline Dependiente & $\operatorname{csd}$ & $\begin{array}{c}\text { Efectos } \\
\text { marginales }\end{array}$ & $\operatorname{csd}$ & $\begin{array}{c}\text { Efectos } \\
\text { marginales }\end{array}$ \\
\hline Constante & $\begin{array}{c}-4.794^{* * *} \\
(0.234)\end{array}$ & & $\begin{array}{c}-4.799^{* * *} \\
(0.237)\end{array}$ & \\
\hline iia & $\begin{array}{c}0.051^{* * * *} \\
(0.017)\end{array}$ & 0.0175 & & \\
\hline iia2 & $\begin{array}{l}-0.001^{*} \\
(0.001)\end{array}$ & & & \\
\hline iiu & & & $\begin{array}{c}0.00003^{* * *} \\
(9.39 \mathrm{e}-6)\end{array}$ & $9.91 \mathrm{E}-06$ \\
\hline iiu2 2 & & & $\begin{array}{c}-7.54 \mathrm{e}-10 * * * \\
(2.43 \mathrm{e}-10)\end{array}$ & \\
\hline ppr 2010 & $\begin{array}{c}0.009^{* * *} \\
(0.002)\end{array}$ & 0.003 & $\begin{array}{c}0.009 * * * \\
(0.002)\end{array}$ & 0.0031 \\
\hline ppr 20102 & $\begin{array}{c}-7.35 e-6 * * * \\
(1.53 e-6)\end{array}$ & & $\begin{array}{c}-7.59 \mathrm{e}-6^{* * * *} \\
(1.47 \mathrm{e}-6)\end{array}$ & \\
\hline edu & $\begin{array}{c}0.756^{* * *} \\
(0.038)\end{array}$ & 0.2946 & $\begin{array}{c}0.748^{* * *} \\
(0.039)\end{array}$ & 0.2921 \\
\hline idel & $\begin{array}{c}-2.806^{* * *} \\
(0.257)\end{array}$ & -1.0937 & $\begin{array}{c}-2.809 * * * \\
(0.257)\end{array}$ & -1.097 \\
\hline gpubp & $\begin{array}{c}0.00007^{* * *} \\
(0.00001)\end{array}$ & 0.00003 & $\begin{array}{c}0.00008^{* * *} \\
(0.00002)\end{array}$ & 0.00003 \\
\hline Muestra & 2080 & & 2080 & \\
\hline $\mathrm{R}^{2} \mathrm{McF}$ & 0.48 & & 0.48 & \\
\hline$R^{2} p$ & 83.8 & & 83.8 & \\
\hline
\end{tabular}

Fuente: Elaboración propia.

Se estimaron 2 ecuaciones para probar el efecto que la inclusión financiera tiene sobre la cohesión social. En ambos casos el modelo como un todo resultó estadísticamente significativo en términos del estadístico de razón de verosimilitud $(\mathrm{RV})^{1}$ y su capacidad de predicción puede considerarse alta en términos

\footnotetext{
1 Prueba de Razón de Verosimilitud. La hipótesis nula es que todos los coeficientes, sin incluir la constante del modelo, que acompañan a las variables explicativas, son simultáneamente igual a cero.
} 
del estadístico $R^{2} p$, el cual en ambos casos pasa del 83 por ciento de aciertos. ${ }^{2}$ En cuanto al coeficiente de determinación de McFadden $\left(R^{2} M c F\right)$ fue de 48 por ciento en ambos modelos, ${ }^{3}$ lo cual se considera un ajuste moderadamente bueno.

Los coeficientes estimados para todas las variables explicativas fueron estadísticamente significativos, por su parte, los signos de dichos coeficientes prácticamente en todos los casos fueron los esperados de acuerdo a la teoría, excepto en el caso del ingreso, para el cual los signos esperados son contrarios a los obtenidos.

Los efectos marginales ${ }^{4}$ de las variables explicativas, evaluados en las medias de éstas, arrojan resultados significativos, que se interpretan a continuación. Un punto más de acceso al sistema financiero formal, por cada 10,000 habitantes, aumentaría en 1.75 por ciento la probabilidad de que el municipio alcance un alto grado de cohesión social, mientras que, por el lado del uso del sistema, un aumento de 1000 cuentas más por cada 10,000 habitantes, aumentaría esta probabilidad en casi 1 por ciento. Igualmente, el aumento de 1,000 pesos reales en el PIB per cápita anual, en ambos modelos aumentaría en 0.3 por ciento la probabilidad de contar con una alta cohesión social. En educación, un aumento promedio de 1 año más de preparación entre los jefes de hogar, aumentaría la probabilidad de alcanzar una alta cohesión en casi 30 por ciento en ambas ecuaciones. Por su parte, tanto en la ecuación estimada 1, como la 2, el aumento de 1 por ciento en el índice de diversidad poblacional, reduciría en 1.09 por ciento esta probabilidad. Finalmente, un aumento de 100 pesos reales en el gasto público per cápita, aumentaría en 0.3 por ciento la probabilidad de que el municipio alcance una alta cohesión social.

La interpretación de los efectos esperados de cambios en las variables de inclusión financiera sobre la cohesión social, pueden también precisarse al calcular sus puntos críticos. Siguiendo la definición de CONEVAL, las ecuaciones estimadas 1 y 2 , mostradas del cuadro 3 , permiten deducir los niveles máximos, donde la inclusión financiera maximiza la probabilidad de que el municipio alcance una alta cohesión social. En la ecuación 1, el número de puntos de acceso que maximiza la probabilidad de una alta cohesión es de 21.5, y en la ecuación 2, el número de contratos que maximiza esta probabilidad es de 22,679.5, ambas medidas por cada 10,000 habitantes en el municipio.

El comportamiento de esta probabilidad, a lo largo del recorrido de las variables de inclusión financiera, puede apreciarse mejor en las gráficas 2 y 3 .

\footnotetext{
2 Coeficiente de determinación de la predicción. Proporción de las observaciones en la muestra que el modelo estimado clasifica correctamente. El valor va de 0 a 1 , entre mayor sea mejor la bondad de ajuste.

3 Coeficiente de determinación de McFadden. $1-L_{1} / L_{0}$, donde $L$ es el logaritmo de la función de verosimilitud, el 0 corresponde al modelo con solo una constante y el 1 al modelo completo (no restringido). Este estadístico, que toma valores de 0 a 1 , no tiene interpretación clara, en especial, no tiene la misma interpretación que en el modelo de regresión lineal estimado por mínimos cuadrados ordinarios.

4 Se puede calcular un efecto marginal para cada observación en la muestra y obtener la media de estos valores. No obstante, como aquí se reportan (cuadro 3), también es posible calcular un efecto marginal evaluando en las medias muestrales de las variables. Los resultados son parecidos, pero no idénticos.
} 
Gráfica 2. Inclusión financiera por acceso y su efecto en la probabilidad de alta cohesión social.

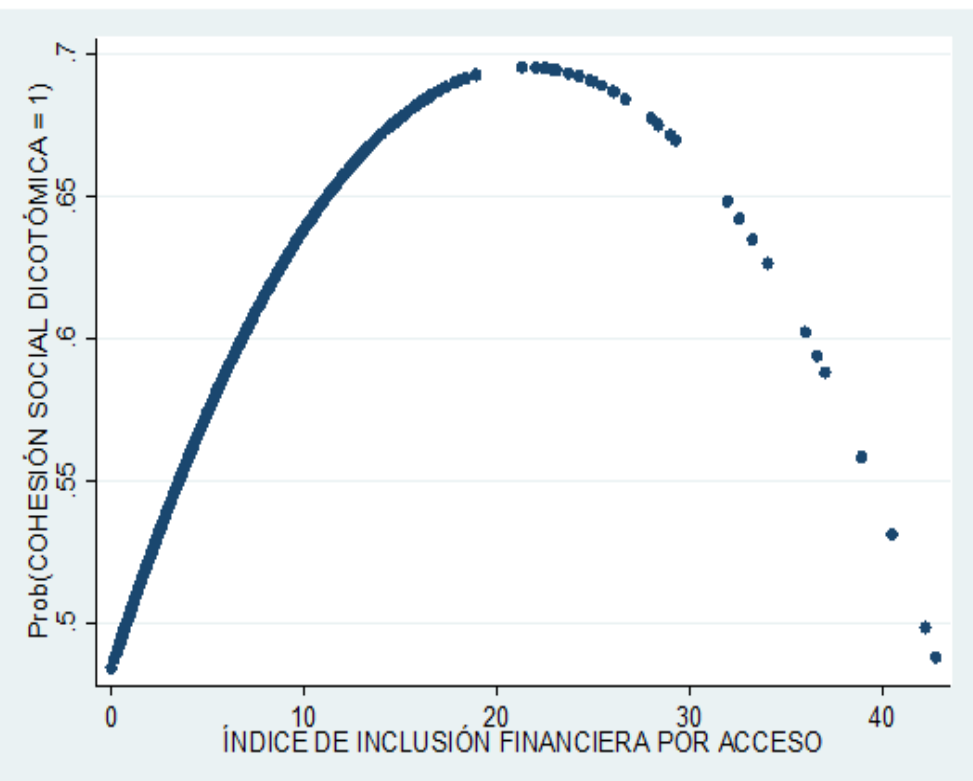

Fuente: Elaboración propia.

Gráfica 3. Inclusión financiera por uso y su efecto en la probabilidad de alta cohesión social.

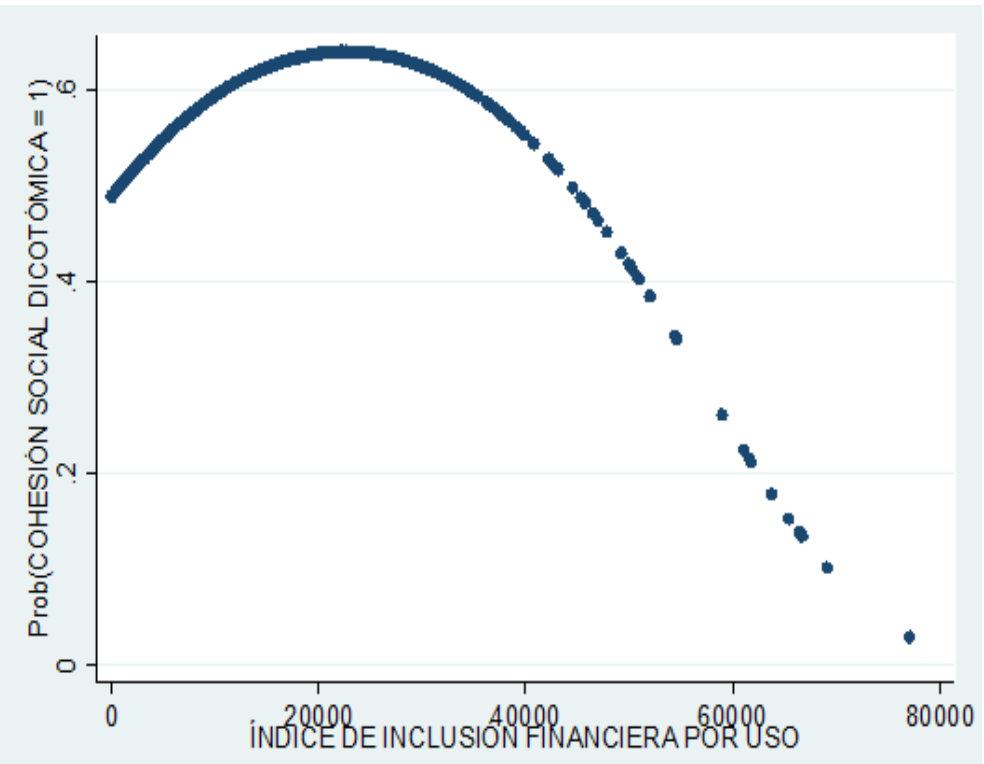

Fuente: Elaboración propia. 
Los puntos críticos de inclusión financiera, por acceso o por uso, aún lucen lejos de ser alcanzados ante la situación en 2010, donde los promedios municipales se encontraron en 3 y 6135 respectivamente (ver cuadro 2). En dicho año, un 95 por ciento de los municipios en México tenían una media de puntos de acceso igual o menor a 11, por su parte, un 90 por ciento de los municipios alcanzaba un total de 21,043 contratos o menos. Esto muestra aún una amplia posibilidad de provocar cohesión social a través del canal de la inclusión financiera, independientemente de lo que pase con el ingreso, la educación, la diversidad poblacional y el propio gasto público, variables que bien podrían considerarse con menores márgenes de maniobra en el ámbito de la política pública en el corto plazo.

Dada la alta concentración de la captación y el crédito financieros en el Distrito Federal, se realizaron todas las estimaciones eliminando los datos correspondientes a las delegaciones de esta entidad federativa. Los resultados no mostraron cambios ni en los signos de los coeficientes, ni en la significancia estadística de los mismos, por su parte la variación en magnitudes también fue mínima. Se realizaron también las estimaciones incluyendo el término de interacción entre el índice de inclusión y el de acceso, resultando dicha variable no significativa, dentro de modelos donde el resto de los coeficientes conservó signo y significancia al comparar con los modelos sin este término de interacción.

Como se señaló en el apartado teórico, el modelo de Gurley y Show (1955) observa a la inclusión financiera como un determinante del desarrollo económico, concepto más amplio que el sólo crecimiento del ingreso nacional. En el presente estudio, el concepto de cohesión social se contempla como un elemento del desarrollo económico y social, tal como lo observa la LGDS. Los resultados estadísticos indicarían que en el caso mexicano se está cumpliendo aquello inicialmente propuesto por Gurley y Shaw (1955), que luego fue ampliado por Bencivenga y Smith (1998). También debe reconocerse, que el presente estudio no dispuso de suficiente información para probar la posible mutua dependencia entre inclusión financiera y cohesión social, lo cual queda como una línea de investigación, para trabajos futuros.

\section{Conclusiones}

El 3 de octubre de 2011 se creó en México el Consejo Nacional de Inclusión Financiera, con el objetivo de formular y poner en marcha la Política Nacional de Inclusión Financiera, como medio para acceder a una mayor inclusión y cohesión social. Dicha política fue aprobada el 15 de junio de 2016 por el Consejo Nacional de Inclusión Financiera (CONAIF, 2016). El presente estudio estimó el efecto que la inclusión financiera ejerce sobre la cohesión social en los municipios de México.

A la luz de los planteamientos teóricos clásicos de cohesión social (Luckmann, 1996, Benger, 1999, Granovetter, 1973), y las propuestas teóricas económico-financieras de Gurley y Shaw (1955), Benvcivenga y Smith (1998) y Makesh y Kuzhuvelil (2014) sobre la influencia de la inclusión financiera en la inclusión social y de los conceptos de inclusión financiera de CONAIF (2015) y de cohesión social de CONEVAL $(2015$, b), las estimaciones realizadas con datos de SIMBAD-INEGI y CNBV (2015), permitieron obtener evidencia estadística de una relación no lineal y estadísticamente significativa entre ambas variables, controlando para otros determinantes de la cohesión social, estos 
últimos encontrados en la revisión de literatura de corte empírico.

Los resultados de la estimación permiten evidenciar que la política encaminada a aumentar la inclusión financiera tiene una alta probabilidad de propiciar una mayor cohesión social en el corto plazo y por varios años y con ello propiciar mejores condiciones de vida entre la población mexicana, principalmente en los municipios donde la inclusión financiera fue tradicionalmente baja o inexistente.

Las medias de puntos de acceso y uso del sistema financiero en los municipios de México se encontraban en 2010 aún alejadas de los niveles que llevarían a maximizar la probabilidad de que los municipios alcancen una alta cohesión social. Los datos más recientes sobre índices de inclusión financiera, correspondientes a la Encuesta Nacional de Inclusión Financiera, INEGI-CNBV 2015, han permitido constatar un aumento significativo en el uso de servicios financieros y crédito entre los adultos mexicanos, pero aún distan de llegar a los puntos críticos obtenidos en el presente estudio, sobre todo al hablar del número de contratos entre el sistema y los usuarios, en la mayor parte del país, donde los promedios estatales más altos alcanzados en 2015, apenas representan una tercera parte del nivel obtenido al deducir los puntos críticos, empleando las ecuaciones aquí estimadas. Ello refuerza la recomendación de seguir ampliando los niveles de inclusión financiera, tanto por el lado del acceso al mismo, como del uso de éste.

El estudio contempló información de 2080 de los 2456 municipios de México. Este número de observaciones, obedeció a que para algunas de las variables que intervienen en las estimaciones no se contó con datos para el 100 por ciento de los municipios mexicanos, esto es una limitación de la presente investigación. Estimaciones con mayor número de observaciones y para años más recientes serían muy útiles para confirmar los resultados aquí obtenidos. Igualmente, dicha información, en conjunto con mediciones más generalizadas, sobre la cohesión social a nivel vecinal o por colonia, permitirían también verificar otras importantes caras del fenómeno y extender su comprensión hacia los efectos que conlleva sobre otras variables sociales y económicas, objetivo de las diferentes políticas públicas.

Siguiendo el modelo teórico seminal de Gurley y Shaw (1955), convendría probar la posible mutua influencia entre inclusión financiera y cohesión social. El desarrollo de nuevos instrumentos de ahorro y crédito, que contemplan la figura de grupos solidarios y microcréditos a través de canales no tradicionales, como son los dispositivos electrónicos, cada vez más presentes en los rincones alejados del país, bien podrían significar la posibilidad de una mayor inclusión financiera, como efecto de una mayor cohesión social.

Finalmente, la presente investigación de corte empírico, da pauta para considerar la posible ampliación del modelo de Gurley y Shaw (1955), ya que el caso mexicano bien puede ser representativo de lo que actualmente está sucediendo en otras partes del mundo, donde la inclusión financiera está en marcha y cuenta con amplias posibilidades de seguir creciendo a la par de las innovaciones tecnológicas y la competencia de nuevos jugadores en el campo financiero. 


\section{Bibliografía}

Acemoglu, D. \& Robinson, J. A. (2013). ¿qué fracasan los países?. México: Crítica.

Almeida, E. \& Sánchez, M. (2014). Comunidad: Interacción, conflicto y utopía. México: Universidad Iberoamericana, ITESO y BUAP.

Arcand, J. L., Berkes, E. \& Panizza, U. (2012). Too Much Finance? IMF Working Paper 12/161, International Monetary Fund Washington, DC. Disponible en https://www.imf. org/external/pubs/ft/wp/2012/wp12161.pdf Banco de México (2011). Circular 14/20

11. Disponible en http://www.banxico.org.mx/disposiciones/circulares/circular-2019$95 /$

Bencivenga, V. \& Smith, B. (1998). Economic Development and Financial Depth in a Model with Costly Financial Intermediation. Research in Economics. 52(4), pp. 363-386.

Berger, P. (1999). Los límites de la cohesión social. Barcelona: Galaxia Gutenberg y Círculo de Lectores.

Bilbao-Ubillos, J. (2013). The limits of Human Development Index: The Complementary Role of Economic and Social Cohesion, Development Strategies and Sustainability. Sustainable Development, 21(6), pp 400-412.

Bolvinik, J. (2010). Principios de la medición multidimensional de la pobreza. En J. Boltvinik, S. Chakravarty, J. Foster, D. Gordon, R. Hernández, H. Soto y M. Mora (Coordinador), Medición multidimensional de la pobreza en México (pp. 43-279). México: El Colegio de México; Consejo Nacional de Evaluación de la Política de Desarrollo Social.

Cámara de diputados del H. Congreso de la Unión (2013). Ley General de Desarrollo Social. Disponible en http://www.diputados.gob.mx/LeyesBiblio/pdf/264.pdf

Comisión Económica para América Latina (2007). Cohesión social: inclusión y sentido de pertenencia en América Latina y el Caribe. Santiago de Chile: CEPAL.

Collins, D., Morduch, J., Rutherford, S., y Ruthven, O. (2011). Las finanzas de los pobres. México: Debate.

Comisión intersecretarial para la prevención social de la violencia y la delincuencia (2013). Programa nacional para la prevención social de la violencia y la delincuencia. Disponible en http://www.gobernacion.gob.mx/archivosPortal/pdf/Bases120213.pdf

Comisión Nacional Bancaria y de Valores, CNBV. (2015). Base de datos de ahorro financiero y financiamiento a nivel municipal. Disponible en http://portafoliodeinformacion.cnbv. gob.mx/estudios/Paginas/default.aspx

Consejo Nacional de Inclusión Financiera, CONAIF. (2015). Qué es la inclusión financiera? Disponible en http://www.cnbv.gob.mx/Inclusi\%C3\%B3n/Paginas/Descripci\%C3\%B3 n.aspx Consejo Nacional de Inclusión Financiera, CONAIF. (2016). Comunicado de Aprobación de la Política Nacional de Inclusión Financiera. Disponible en http://www. cnbv.gob.mx/PRENSA/Prensa\%20\%20Otros/Comunicado\%20Conjunto\%20Politica \%20Nacional\%20de\%20Inclusion\%20Financiera.pdf

Consejo Nacional de Evaluación de la Política de Desarrollo Social. CONEVAL. (2015). Medición de la pobreza en México y en las entidades federativas 2014. Disponible en http://www.coneval.gob.mx/Medicion/Documents/Pobreza

Consejo Nacional de Evaluación de la Política de Desarrollo Social. CONEVAL. (2015, b). Medición de la pobreza: Cohesión social. Disponible en http://www.coneval.gob.mx /Medicion/Paginas/Cohesion_Social.aspx

Chan, J., Hong-Po, T., \& Chan, E. (2006). Reconsidering social cohesion: Developing a definition and analytical framework for empirical research. Social Indicators Research, $75(2)$, pp. $273-302$.

Durkheim, E. (1993). La división del trabajo social. México: Colofón.

Easterly, W., Ritzen, J., \& Woolcock, M. (2006). Social cohesion, institutions, and growth. Economic \& Politics, 18(2), pp. 103-120.

European Commission (2010). The Strategy Europe 2020. Disponible en http://ec.europa.eu /europe2020/index_en.htm 
Foster, J. (2010). Informe sobre la medición multidimensional de la pobreza. En J. Boltvinik, S. Chakravarty, J. Foster, D. Gordon, R. Hernández, H. Soto y M. Mora (Coordinador), Medición multidimensional de la pobreza en México (pp. 323-399). México: El Colegio de México y Consejo Nacional de Evaluación de la Política de Desarrollo Social.

Fromm, E. (2010). El miedo a la libertad. México: Paidós.

Fuentes, M. (2008). Complejidad y exclusión social. En R. Cordera, P. Ramírez y A. Ziccardi (Coords.), Pobreza, desigualdad y exclusión social en la ciudad del siglo XXI, (pp. 182-196). México: UNAM, Instituto de Investigaciones Sociales, Siglo XXI.

Garrido, A. y Álvaro, J. (2007). Psicología social. España: McGraw-Hill/Interamericana.

Goetzmann, W. (2016). Money changes everything: How finance made civilization possible. New Jersey: Princeton University Press.

Gradstein, M. \& Justman, M. (2002). Education, Social Cohesion, and Economic Growth. American Economic Review, 92(4), pp. 1192-1204.

Granovetter, M. (1973). The Strength of Weak Ties. American Journal of Sociology, 78(6), pp. $1360-1380$.

Granovetter, M. (1983). The strength of weak ties: A network theory revisited. Sociological Theory, 1, pp.201-233.

Granovetter, M. (2005). The Impact of Social Structure on Economic Outcomes. Journal of Economic Perspectives, 19(1), pp. 33-50.

Greene, W. (2012). Econometric Analysis. England: Pearson.

Greenwood, J. \& Jovanovic, B. (1990). Financial Development, Growth, and the Distribution of Income. Journal of Political Economy, 98(5), pp. 1076-1107.

Gurley, J. \& Shaw, E. (1955). Financial aspects of Economic Development. American Economic Review, 45(4), pp. 515-538.

Institute for Digital Research and Education - University of California, Los Angeles. (IDREUCLA). (2015). Stata Data Analysis Examples Probit Regression. Disponible en http://www.ats.ucla.edu/stat/stata/dae/probit.htm

Inoue, S., Yorifuji, T., Takao, S., Doi, H., \& Kawachi, I. (2013). American Journal of Public Health, 103(12), 60-66.

Instituto Mexicano para la Competitividad, A.C. (2014). ¿Quién manda aquí?: La gober nanza de las ciudades y el territorio mexicano. Disponible en http://imco.org.mx/wp content/uploads/2014/09/20140909_LibroCompleto_Indice_de_Competitividad_Urbana_ 2014.pdf

Instituto Nacional de Estadística y Geografía (INEGI) y Comisión Nacional Bancaria y de Valores (CNBV). (2015). Encuesta Nacional de Inclusión Financiera, 2015. Disponible en http://www.inegi.org.mx/est/contenidos/proyectos/encuestas/hogares/especiales/ enif/enif2015/default.aspx

Kempson, E., Whyley, C., Caskey, J., \& Collard, S. (2000). In or out? Financial exclusion: a literature and research review. Bristol, Financial Services Authority. Diponible en http://www.pfrc.bris.ac.uk/Reports/In_or_out.pdf

Kuznets, S. (1955). Economic Growth and Income Inequality. The American Economic Review, 45(1), pp. 1-28.

Lipovetsky, G. (2016). De la ligereza. Barcelona, Barcelona: Anagrama.

Luckmann, T. (1996). Teoría de la acción social, Barcelona: Paidós.

Makesh, K. G. \& Kuzhuvelil, M. (2014). Financial inclusion: A Literature on its Causes and Effects. International Journal of Applied Financial Management Perspectives, 3(1), pp. 745-750.

Martínez, P. y Estefanía, J. (Coords.) (2016). América Latina: un nuevo contrato social. España: Centro de estudios Iberoamericanos Jesús Polanco. Disponible en: http://www.marcialpons.es/static/pdf/9788491231592.pdf México Evalúa y Fundación Hogares (2015). Bases para la construcción de un índice de cohesión social vecinal. Disponible en http://mexicoevalua.org/2015/09/bases-para-la-construccion-de-un-indi ce-de-cohesion-social-vecinal/

Oxoby, R. (2009). Understanding Social Inclusion, Social Cohesion and Social Capital. Economic Research Paper: 2009-09. Department of Economics, University of Calgary. Diponible en http://lcerpa.org/public/papers/LCERPA_2009-09.pdf 
66 Nueva Época REMEF (The Mexican Journal of Economics and Finance)

Peña, X., Hoyo, C. y Tuesta, D. (2014). Determinantes de la inclusión financiera en México a partir de la ENIF 2012. Disponible en https://www.bbvaresearch.com/wpcontent/uploads/2014/06/WP_1414.pdf

Putnam, R. (2000). Bowling alone: The collapse and revival of American community. New York: Simon \& Schuster.

Ratna, S., Cihák, M., NDiaye, P., Barajas, A., Bi, R., Ayala, D., Gao, Y., Kyobe, A., Nguyen, L., Saborowski, Ch., Svirydzenka, K. \& Reza, S. (2015). Rethinking Financial Deepening: Stability and Growth in Emerging Markets. IMF Staff Discussion Note 15/08, International Monetary Fund Washington, DC. Diponible en https://www.imf.org/external /pubs/ft/sdn/2015/sdn1508.pdf

Rodríguez, D. y López, F. (2009). Desarrollo financiero y crecimiento económico en México. Revista latinoamericana de economía, 40(159), pp. 39-60.

Sánchez, K. (2012). Social exclusion, social cohesion: Defining narratives for development in Latin America. Journal of International Development, 24(6), pp. 728-744.

Salazar, J., Jaramillo, J. \& Álvarez, B. (2015). Financial inclusion and income inequality in Mexican municipalities. Open Journal of Social Sciences, 3(12), 29-43.

Timasheff, N. (2006). La teoría sociológica, México: Fondo de Cultura Económica.

Vargas, D. y Merino, M. (2012). El papel de los espacios públicos y sus efectos en la cohesión social: experiencia de política pública en México. Estudios Sociológicos, 30(90), 897-914.

Venegas, F., Tinoco, M. \& Torres, V. (2008). Deregulation, financial development and economic growth in Mexico: Long-term effects and causality. SSRN. Disponible en https://papers.ssrn.com/sol3/papers.cfm?abstract_id=1010525

Zavaleta, O. y Urbina, H. (2011). Mercados financieros y desarrollo económico: un análisis sobre México. Ide@s CONCYTEG, 6(68), 226-235. Disponible en http://www.concyteg .gob.mx/ideasConcyteg/Archivos/68032011_MERCADOS_FINANCIEROS_DESARRO LLO_ECONOMICO.pdf 